\title{
TRANSMISSION OF CHOSEN TRANSFORM COEFFICIENTS OF NORMALIZED CARDIAC BEATS FOR COMPRESSION
}

\author{
S. Saha $\quad$ A. G. Ramakrishnan, Senior Member IEEE \\ Department of Electrical Engineering, Indian Institute of Science, Bangalore 560 012, INDIA
}

\begin{abstract}
A new technique for $\mathrm{ECG}$ compression is presented. Each delineated ECG beat is period normalized by multirate processing and then amplitude normalized. Dis crete Wavelet Transform (DWT), based on Daubechies-4 basis functions is applied on these normalized beats, after shifting each of them to the origin. The concatenation of ordered DWT coefficients of these beats is a near-cyclostationary signal. An algorithm is proposed to select a set of common positions of the significant coefficients to be retained from each beat. Iinear Prediction is then applied to predict only these DWT coefficients of the current beat from the corresponding coefficients of a certain number of previous beats. Transmitting only the residuals of selected coefficients improves compression. A significant advantage of this technique is that the maximum reconstruction error in any cycle does not occur in the diagnostically crucial QRS region, while achieving a compression of about 15:1 and a normalized root mean square error of about $10 \%$.
\end{abstract}

\section{INTRODUCTION}

The need for ECG coding arises in several contexts such as ambulatory ECG monitoring, patient data bases in major hospitals, medical education systems, cost effective transmission of ECG over telephone lines, etc. ECG belongs to a class of signals that are oscillatory in nature, although not periodic in the strict mathematical sense. By looking at the time evolution of these signals, a concatenation of similar events or periods can be observed, which almost never identically reproduce themselves. For the same subject, the cycle to cycle variation in the beat period is, in general, much higher than the occasional variations in the amplitude, position, and width of the constituent waves. However, most techniques of ECG compression reported till now have not exploited this correlation between cycles (inter-beat correlation). There is also some redundancy within each ECG cycle. Direct time-domain techniques (such as AZTEC and SAPA) and transform-domain techniques [1] have considered only this intra- beat correlation between successive samples. Whereas, long term prediction (2) and average beat subtraction [3] techniques have used only the beat to beat correlation, ignoring the redundancy within beat. Another limitation of [2] and [3] arises from the fact that, since the period of a beat changes constantly, points that are equidistant but farther from the $\mathrm{R}$ wave in two different cycles are not always well correlated. Further, [2] requires detection of the end points of each cycle, in addition to QRS detection and the correlation of QRS complex of each beat with a codebook of representative complexes. Similarly, the methods based on modeling require component identification for both model order selection and proper cycle separation. Parametric techniques have minimized only the intra-beat redundancy and not the other.

The technique proposed in this paper accounts for the variations in the beat periods and then exploits both the inter-cycle and the intra-cycle correlations. The method requires the detection of no component other than the Rwave. We first make the period of each beat constant by multirate processing, a nearly reversible transformation in which the original periods can be restored without any loss. By the term 'period', we mean the number of samples between two consecutive $\mathbf{R}$ waves. We perform amplitude normalization on these period- normalized beats, to bring about greater similarity.

On these 'period and amplitude normalized' (PAN) beats, Discrete Wavelet Transform (DWT) is applied. As a result, the corresponding wavelet coefficients (across cycles) bear a lot of correlation. Linear prediction of the wavelet coefficients trom past cycles has been performed to effectively utilize this correlation. This enables us to transmit only the prediction error of the wavelet coefficient sequence, which has a lower dynamic range than the original sequence. The DWT has been applied to ECG for compression by Bradie [4] and Thakor (5). However, in [4], the similarity of corresponding DWT coefficients across the beats could not be exploited. This was because, unlike our approach, padding of zeros was done to the variable period beats to make the periods uniform. In (5), the authors have not clearly specified the beginning and end points of sequences whose DWT they compute. Orr method has been tested on both normal and abnormal data and its performance evaluated using several measures of fidelity.

\section{IMPLEMENTATION OF PAN}

Individual constant period vectors are formed from each ECG cycle. We used the technique reported in (5) for QRS detection. We normalize the period of each isolated 
beat by multirate signal processing techniques $(6)$. This essentially involves sampling rate change by different fractional factors for different cycles. This converts the beats of differing periods into beats of a constant period, thus eliminating the effect of heart rate variability. The fixed length of the cycles is selected based on the maximum possible period of any cardiac cycle and the sampling frequency. The modified sampling rate must still satisfy the Nyquist criterion. We have selected a length such that the new sampling rate is always higher than the original one, ensuring that there will be no distortion of the signal. The mean beat period (MBP) is estimated from some initial cycles of the data being coded. This value is initially sent to the decoder. During encoding, the difference between the actual period of a cycle and the MBP is transmitted.

To perform period-normalization, we first interpolate the variable period beat vectors by a factor $L$, which is the fixed period planned for (the length of the normalized beat). Then the signal is downsampled by the appropriate factor for each cycle, so that the length of all cycles becomes uniform. In our case, only the interpolation filter and downsampling are required. Since ECG is a highly correlated signal, and since it has been interpolated by a sufficiently high value, no error occurs in downsampling. The details of implementation are given below.

If $x(n)$ is the input to an interpolation filter with an upsampling factor $L$ and an impulse response $b(\mathbf{n})$, then the output $y(\mathbf{n})$ is given by

$$
y(n)=\sum_{k=-\infty}^{\infty} x(k) h(n-k L)
$$

The upsampler simply inserts $L-1$ zeroes between successive samples. The filter $h(n)$, which operates at a rate $\mathrm{L}$ times higher than that of the input signal, replaces the inserted zeroes with interpolated values. Polyphase implementation of this filter (7) ensures efficient interpolation. The output $y(n)$ of a decimation filter, with an impulse response $\boldsymbol{h}(0)$ and a downsamplina factor $M$, is given by

$$
y(n)=\sum_{k=-\infty}^{m} x(k) h(n M-k)
$$

where $b(a)$ is a low-pass filter used to remove the aliasing caused by the downsampling of the signal. In case the signal does not contain trequencies above $\pi / M$, there is no need for the decimation filter; downsampling by a factor of $M$ will do. The change of sampling rate thus achieved is a reversible process, provided Nyquist condition is satisfied; if the resampled beat vector is brought back to the original sampling rate by multirate processing, there will be no distortion. The output of our system is thus given by

$$
\mathrm{Y}_{i}(n)=\sum_{k=0}^{P_{i}-1} \mathrm{X}_{i}(k) h\left(n M_{i}-k L\right)
$$

where $X_{i}(n), Y_{i}(n)$ are the $n^{\text {th }}$ samples of the $i^{\text {th }}$ input beat and output PAN beat respectively, $h(n)$ is the impulse response of the multirate filter and $\boldsymbol{P}$, is the total No. of samples in the $i^{\text {th }}$ original beat.
Amplitude normalization brings about further similarity between the beat patterns. Each sample of a timenormalized beat is divided by the magnitude of the sample having maximum amplitude in that particular beat. This makes the highest amplitude sample(s) of each beat equal to unity. Thus the variations between the magnitudes of different cycles are minimized and the intra-cycle correlation is enhanced. To begin with, the average amplitude scale factor (AASF) is obtained from a few initial beats and is sent to the decoder. Subsequently, for each cycle being coded, the difference between the maximum amplitude of that cycle and the AASF is transmitted to the decoder.

\section{PREDICTION OF DWT COEFFICIENTS}

It is on this PAN beat, that we apply the DWT . The reason for applying the DWT is the highly non-stationary nature of any ECG beat. The QRS complex of ECG is a well-localized, high-frequency region. $\mathrm{P}$ and $\mathrm{T}$ waves are low trequency components, while the $\mathrm{PQ}$ and TP segments are nearly iso-electric with no clinically useful information and the ST segment is a very low frequency, well time-localized component of ECG.

Of the many available functions $\Psi_{k, k}(x) \in \mathbf{L}^{2}(\mathbf{R})$, which satisfy orthonormality, we have used Daubechies-4 (D4) wavelet basis functions for representing each PAN beat. The D4 wavelet $\Psi_{0,0}(\boldsymbol{x})$ occupies three unit intervals $0 \leq x<3$, which are wrapped around $0<x<1$ for signal representation. The expansion of any given signal $f(x)$ in $0 \leq x \leq 1$ can be written $\mathbf{a s}(7)$,

$$
\begin{aligned}
f(x)= & w_{01} \phi(x)+w_{11} \Psi(x)+\left[\begin{array}{ll}
w_{21} & w_{22}
\end{array}\right]\left[\begin{array}{c}
\Psi(2 x) \\
\Psi(2 x-1)
\end{array}\right] \\
& +\left[\begin{array}{llll}
w_{31} & w_{32} & w_{33} & w_{34}
\end{array}\right]\left[\begin{array}{c}
\Psi(4 x) \\
\Psi(4 x-1) \\
\Psi(4 x-2) \\
\Psi(4 x-3)
\end{array}\right]+\cdots
\end{aligned}
$$

where $\phi(x)=1,0 \leq x<1$ and $w_{j k}$ refers to the wavelet coefficient at scale $\vec{j}$ and location $\boldsymbol{k}$. A vector is formed by stacking the above wavelet coefficients at scale $0,1,2, \cdot$. for the $j^{\text {th }}$ beat, as defined below:

$$
X_{j}=\left[w_{01}(j) w_{11}(j) w_{21}(j) w_{22}(j) \cdots\right]
$$

To compute the wavelet coefficients for the signal (each PAN beat) sampled at equally spaced intervals in $0 \leq x \leq$ 1 , we use Mallat's Pyramidal algorithm [8]. When applied on a PAN beat, owing to the non-stationary nature of the latter and non-uniform distribution of energy across \&fferent scales, it turns out that only the top $20 \%$ of the coefficients are necessary for good quality reconstruction. Also there is good similarity between the corresponding wavelet coefficients of each beat. So we have performed linear prediction of selected wavelet coefficients across cycles and transmitted the residuals to the decoder.

From $(5)$, if $\mathbf{V}=\left[X_{1} X_{2} \cdots X_{N_{T}}\right]$, then $\mathrm{V}$ is a near cyclo-stationary sequence. Rom such a sequence, by regrouping the corresponding (periodic) coefficients, widesense stationary (WSS) data sets are formed. It has been 
observed that many of the wavelet coefficient sets from the above are not significant in reconstructing the ECG beat (because of the suitability of D4 wavelet bases). Hence, based on their relative magnitudes, some of the above near WSS data sets are selected and independent linear prediction (LP) is performed on these sets. By judiciously choosing a fixed set of locations at which LP needs to be performed, we can eliminate the overhead of bits required to send the positions of retained coefficients. The algorithm used for deciding the set of significant locations of wavelet coefficients to be retained in each cycle as follows:

1. $P_{i}=\left\{p_{i j}\right\}, \begin{aligned} i= & 1,2, \ldots, \mathrm{L} \\ j= & 1,2, \ldots, N_{c} \\ & N_{c} \leq N_{R}\end{aligned}$

where $N_{R}$ is the final number of coefficients to be retained in each beat.

2. Initialize $N=N_{m}<N_{c}$, where $N_{m}$ is the minimum number of highest amplitude wavelet coefficients to - be retained in each beat.

$$
\begin{aligned}
& \text { 3. } P_{i}^{N}=\left\{p_{i j}\right\}, \quad i=1,2, \ldots, L \\
& j=1,2, \ldots, N \text {. }
\end{aligned}
$$

4. $S_{N}=\bigcup_{i=1}^{L} P_{i}^{N}$

5. If $\left|S_{N}\right|=N_{R}$, stop.

6. If $\left|S_{N}\right|<N_{R}, \mathrm{~N}=\mathrm{N}+1$ and go to step 3 .

7. If $\left|S_{N}\right|>N_{R}$, then, from the last set of locations added, remove $\left(\left|S_{N}\right|-N_{R}\right)$ entries corresponding to coefficients of least magnitude.

8. $S_{N}$ is now the set of locations of wavelet coefficients to be retained in every beat.

At the decoder, wavelet coefficients are reconstructed from their residuals. By applying the inverse discrete wavelet transform, the reconstructed PAN beats are obtained. The actual amplitude and period beats are reconstructed by the same process as for PAN, with suitable change of parameters. To assess the quality of the reconstructed signal, we employ different error measures as discussed in the next section.

\section{RESULTS}

The proposed method was tested on ECG data from 24 different subjects obtained from a hospital. The ECG signal was sampled at $250 \mathrm{~Hz}$ and quantized with 12-bitresolution. During period normalization, the length of each ECG cycle was changed to 256 samples. The technique was also applied on five sets of data from MIT database. The performance of the method is evaluated using the Compression ratio (CR), Normalized Root Mean Square Error (NRMSE), Normalized Maximum Amplitude of Error (NMAE) and position of peak reconstruction errors in each cycle.

The expression for the compression ratio for this technique is given below:

$$
C R=\frac{\alpha_{o} \sum_{i=1}^{N_{T}} T_{i}}{N_{T}\left(N_{R} \alpha_{r}+\alpha_{a}+\alpha_{p}\right)+\left\{N_{R} C\right\}}
$$

where

$$
C=\left(p \beta_{l p}+\beta_{n z}\right)+\beta_{m p}+\beta_{m a}
$$

and

$\boldsymbol{\alpha}_{\boldsymbol{0}}$ is the no. of bits per sample in original,

Ti is the period of the $i^{i t h}$ beat,

$N_{T} \quad$ is the total no. of beats processed,

$N_{R} \quad$ is the no. of retained wavelet coefficients,

$\alpha_{r} \quad$ is the no. of bits used for each residual,

$\alpha_{a}$ is the no. of bits used for trans. each SD,

$\alpha_{p}$ is the no. of bits used for trans. the each PD,

$P \quad$ is the order of the prediction filter,

$\beta_{l p}$ is the no. of bits for trans. each LP parameter,

$\beta_{n z}$ is the no. of bits for trans. each element of $S_{N}$,

$\beta_{m p}$ is the no. of bits for trans. the MBP and

$\beta_{m a}$ is the no. of bits for trans. the AASF.

The amplitude of the maximal reconstruction error in each cycle is normalized by the dynamic range of the signal to obtain the NMAE. The expression for NMAE for the $i^{\text {th }}$ cycle is

$$
N M A E_{i}=\frac{\max \left|\mathbf{X}_{o i}-\mathbf{X}_{r i}\right|}{\max \mathbf{X}_{o i}-\min \mathbf{X}_{o i}}
$$

The mean NMAE for a subject is obtained by averaging over all the cycles.

The terms within braces in the denominator of (6) need to be sent to the decoder only once. We have obtained CR of around 18:1 (including overheads), with average NRMSE of $10 \%$ and NMAE of $7 \%$. A significant aspect of our technique is that peak errors do not lie in QRS regions, even while maintaining the overall error within limits. Most of the previous authors have not achieved this, with the exception of Phillips [9\}. Table I gives the performance figures for 6 different subjects. Fig 1 displays the original, reconstructed and error waveforms for a subject.

\section{CONCLUSION}

A novel scheme for high fidelity coding of ECG has been proposed. Handling R-R beats eliminates the ambiguity that normally arises in deciding the endpoints of a cycle whenever PQRST beats are used. The method, based on linear prediction of chosen wavelet coefficients of normalized beats, performs well with both normal and pathological data. The normalization of beats has not been attempted or even conceived of by any other earlier work, even where each beat is taken as an unit, or inter-cycle correlation is exploited $\{2,3)$. This preprocessing converts the ECG data into a near-cyclostationary sequence and enables the uniform choice of wavelet coefficients to be retained in each beat and hence their prediction. The earlier, wavelet-based compression schemes have not attempted this $(4,5)$. With the proposed technique, a mean transmission rate of 180 bits per second has been achieved for the data tested, with no compromise on the fidelity of reconstruction. It exploits both interbeat and intrabeat correlations. Further, the clinically more significant QRS complexes are coded with an error equal to that in the other regions in each cardiac cycle. 


\begin{tabular}{|r|c|r|r|}
\hline Subj. No. & CR & NRMSE\% & NMAE\% \\
\hline $\mathbf{1}$ & 19.1 & 11.27 & 9.77 \\
$\mathbf{2}$ & 22.3 & 13.34 & 6.81 \\
3 & 20.2 & 12.98 & 5.03 \\
4 & $\mathbf{1 1 . 1}$ & 8.49 & 4.96 \\
5 & 9.81 & 7.65 & 5.13 \\
6 & 15.9 & 11.06. & 5.44 \\
\hline
\end{tabular}

\section{References}

[1] G. Nave and A. Cohen, "ECG compression using longterm prediction," IEEE Runs. Biomed. Eng., vol. 38, pp. 235-259, Mar. 1991.

(2) P.S.Hamilton and W.J.Tompkins, "Compression of ambulatory ECG by average beat subtraction and residual differencing," IEEE Runs. Biomed. Eng. vol. 38, pp. 235-259, Mar. 1991.

[3] B. Bradie, "Wavelet packet-based compression of single lead ECG," IEEE Trans. Biomed. Eng. vol. 43, pp. 493-501, 1996.

[4] N. V. Thakor, Y. Sun, H. Rix, and P. Caminal, "MULTWAVE: a wavelet based EC G data compression algorithm," IEICE Trans. Information, System (Japan), E76D: 1462-1469, 1993.

[5] J. Pan and W.J. Tompkins, "A real time QRS detection algorithm", IEEE Trans. Biomed. Eng. vol. 32, pp. 461-465, 1983.

[6] P.P. Vaidyanathan, Multirate systems and Filter banks, Prentice Hall, Englewood Cliffs, 1993.

[7] D.E. Newland, Random Vibrations: Spectral and Wavelet Analysis, John W iley and Sons, 1993.

[8]W. Phillips, "ECG data compression with time warped polynomials," IEEE Runs. Biomed. Eng., vol. 40, pp. 1095-1101, Nov. 1993.

(9)S.M.S. Jalaleddine, C.G. Hutchens, R.D. Strattan, and W.A. Coberly, "ECG data compression techniques - A unified approach," IEEE Runs. Biomed. Eng. vol.37, pp. 329-343, April 1990.

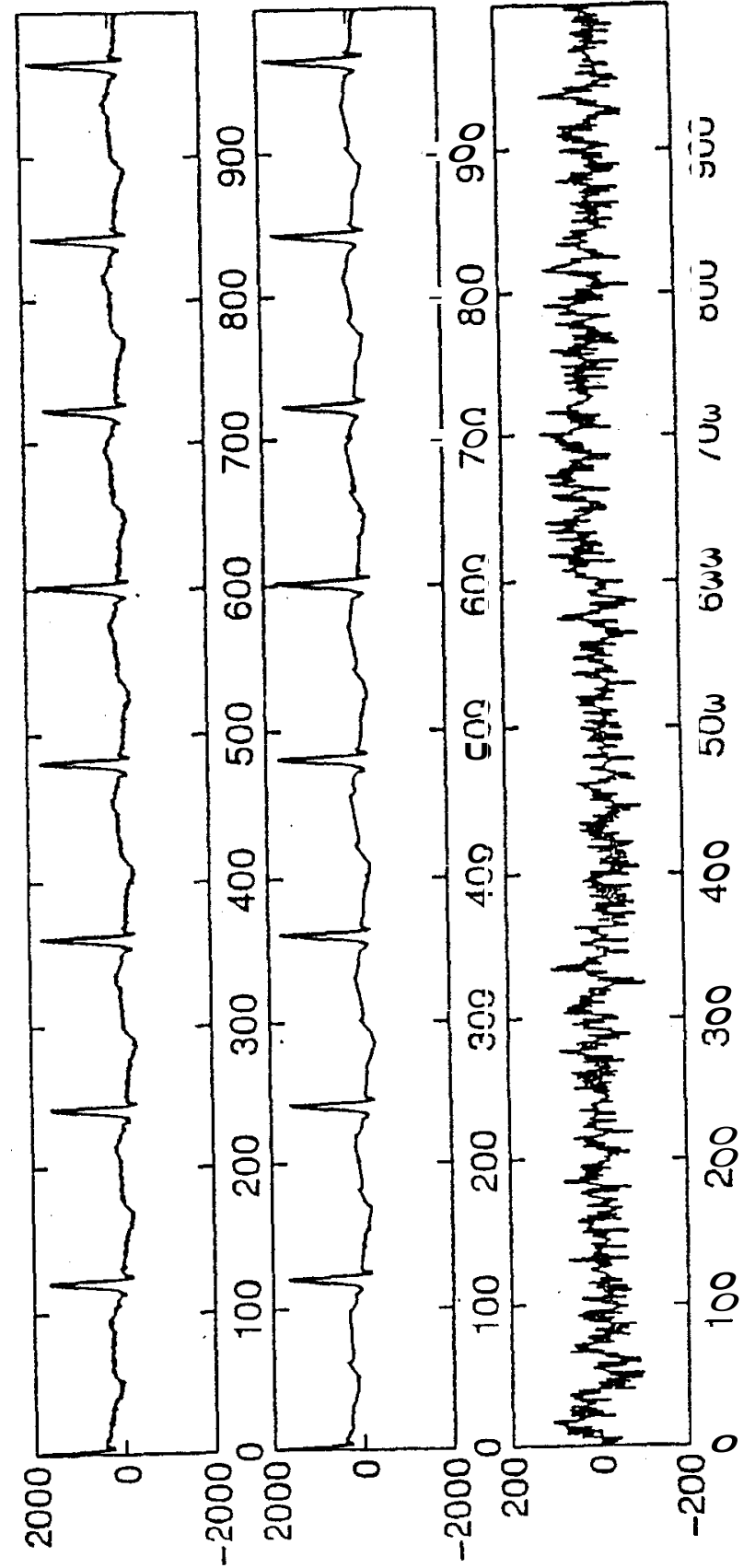

Figure 1. Original ECG. Reconstructed ECG and the reconstruction error (x-axis: sample no. $y$-axis: amplitude) 